\title{
Susceptibility Locus in Neurokinin-I Receptor Gene Associated with Alcohol Dependence
}

\author{
Chamindi Seneviratne', Nassima Ait-Daoud', Jennie Z Ma², Guobo Chen ${ }^{1,3}$, Bankole A Johnson' and Ming D Li*,' \\ 'Department of Psychiatry and Neurobehavioral Sciences, University of Virginia, Charlottesville, Virginia, USA; ${ }^{2}$ Department of Public Health \\ Sciences, University of Virginia, Charlottesville, Virginia, USA; ${ }^{3}$ Institute of Bioinformatics, Zhejiang University, Hangzhou, Zhejiang, People's \\ Republic of China
}

\begin{abstract}
Substance P (SP), a neurotransmitter in stress pathways, exerts its effects mainly through the neurokinin-I receptor (NKIR). Genetic and pharmacological studies show that binding of ligands to NKIR decreases anxiety-related behaviors, and therefore, self-administration of alcohol in mice and craving for alcohol in humans. As genetic variants may result in differential expression of the receptor through various molecular mechanisms, we examined whether allelic variations in the NKIR gene are associated with alcohol dependence (AD) by genotyping II single-nucleotide polymorphisms (SNPs) across NKIR in alcoholic $(n=27 \mathrm{I})$ and healthy control $(n=337)$ participants of Caucasian descent. The AD was diagnosed using the Structured Clinical Interview for Diagnostic and Statistical Manual of Mental Disorders, fourth edition. Associations of the SNPs with AD were assessed at both the individual SNP and haplotype levels. We found that genotype and allele frequencies of rs6715729, a synonymous SNP in exon I, differed significantly in alcoholics and in controls $\left(p=0.0006 ;\right.$ OR (odds ratio) $=6.13 ; 95 \% \mathrm{Cl}=4.06,9.23$ ). Haplotype analyses indicated two risk haplotypes for AD in the $5^{\prime}$ end of the gene, formed by the three-SNP combinations rs67I5729-rs735668-rs674I029. Taken together, we conclude that polymorphisms of NKIR are significantly associated with the development of AD in Caucasian individuals. Additional studies are needed to replicate these results in other samples and to elucidate the mechanism(s) by which these polymorphisms affect NKIR function in the brain. Neuropsychopharmacology (2009) 34, 2442-2449; doi: I0.1038/npp.2009.65; published online 24 June 2009
\end{abstract}

Keywords: association; SNPs; alcohol dependence; addiction; NKIR

\section{INTRODUCTION}

Alcohol dependence (AD) is a psychiatric disorder that is the third most common preventable cause of death and morbidity in the United States (Johnson, 2008). The World Health Organization estimated the number of alcohol users to be 2 billion worldwide in 2002. In an estimate of factors responsible for global disease burden, alcohol contributed $3.2 \%$ of all deaths in 2000 (http://www.who.int/substance _abuse/facts/global_burden/en/).

Despite advances in $\mathrm{AD}$ research, treatment completion and relapse rates remain substantial (Garbutt et al, 2005). The etiology of $\mathrm{AD}$ is complex: once use is initiated, the vulnerability to become dependent on alcohol is modulated by genetic and environmental factors, as well as by their interactions. The contribution of genetic factors to $\mathrm{AD}$ is substantial, with a heritability of 50-64\% (Yang et al, 2007). Thus, it is important to identify genes involved in the

*Correspondence: Professor MD Li, Department of Psychiatry and Neurobehavioral Sciences, University of Virginia, 1670 Discovery Drive, Suite 1 I0, Charlottesville, VA 229| I, USA, Tel: + I 434243 0570, Fax: + I 434973 703I, E-mail: Ming_Li@virginia.edu

Received 7 January 2009; revised 28 April 2009; accepted I I May 2009 neurobiological pathways mediating alcohol's reinforcing effects and associated with its abuse liability.

Dampening of the stress response is one of the main mechanisms by which alcohol exerts its reinforcing effects (Sinha et al, 1998). Animal and human studies suggest a central role for substance P (SP) in mediating the stress response (Holmes et al, 2003). The biological actions of $\mathrm{SP}$ are exerted primarily through neurokinin receptor subtype 1 (NK1R; also called tachykinin receptor 1; TACR1), which is densely expressed in the brain structures implicated in stress pathways (Giegling et al, 2007), as well as in non-neuronal peripheral tissues, including gut, joints, muscle, and cutaneous tissues (Santarelli et al, 2001; Almeida et al, 2004). Antagonists of NK1R are effective in treating depression, anxiety, and post-operative and chemotherapy-induced emesis (Randolph et al, 2004). Recently, a reduction in the craving for alcohol in anxious alcoholics was reported in response to the NK1R blocker, LY686017 (George et al, 2008).

The NK1R protein is encoded by NK1R gene, located on chromosome 2p13.1, spanning about $151 \mathrm{~kb}$ with five exons. According to the NCBI database (http://www.ncbi.nlm.nih. gov), NK1R is transcribed into at least four mRNA variants, resulting from alternative promoter usage, differential splicing, or both. The mRNA variants are putatively 
translated into four functional NK1R isoforms that may possess different affinities for SP. In addition, the expression of these isoforms seems to be tissue specific. For example, so far, only the two longest isoforms of mRNA have been found in the human brain (Caberlotto et al, 2003; Lai et al, 2008). Therefore, naturally occurring polymorphisms at or near promoter or splice regions may determine NK1R density, altering SP-mediated signaling.

The objective of the current study was to determine whether genetic variants of NK1R are associated with AD by performing a case-control association study in a sample of Caucasian $\mathrm{AD}$ and control participants.

\section{MATERIALS AND METHODS}

\section{Participants}

A total of 608 unrelated Caucasian participants were recruited from Central-Southern USA. Participants provided written informed consent on a form approved by all involved institutional review boards. Of these participants, 271 were treatment-seeking persons with AD and 337 were controls. Numbers of participants recruited at each recruitment site are provided in Table 1 . All AD study participants were recruited as part of pharmacotherapy trials for the treatment of AD. AD was diagnosed using the Structured Clinical Interview (First et al, 1994) for Diagnostic and Statistical Manual of Mental Disorders, fourth edition (American Psychiatric Association, 1994) Axis I Disorders. In addition, all the AD study participants had a score of $\geqslant 8$ on the Alcohol Use Disorders Identification Test (AUDIT) (Babor et al, 1992), which assesses the personal and social harm after alcohol consumption, reported drinking of $\geqslant 21$ standard drinks/week for women and $\geqslant 30$ standard drinks/ week for men during the 90 days before enrollment, and negative urine toxicological screen for narcotics, amphetamines, or sedative-hypnotics at enrollment. Abstinence was not an entry criterion. Participants who had current Axis I psychiatric diagnoses other than alcohol or nicotine dependence; significant alcohol withdrawal symptoms (revised clinical institute withdrawal assessment for alcohol scale (Sullivan et al, 1989) score $>15$ ); clinically significant physical abnormalities identified at physical examination, electrocardiogram recording, hematological assessment, biochemistry tests including serum bilirubin concentration and urinalysis; pregnant or lactating state; treatment for $\mathrm{AD}$

Table I Demographics of AD and Control Samples used in this Study

\begin{tabular}{lcc}
\hline & $\begin{array}{c}\text { AD } \\
\text { participants }\end{array}$ & $\begin{array}{c}\text { Control } \\
\text { participants }\end{array}$ \\
\hline Total number & $27 \mid$ & 337 \\
San Antonio, TX & 189 & 4 \\
Charlottesville, VA & 82 & 0 \\
Jackson, MS & 0 & 333 \\
Male/female & $196 / 75$ & $127 / 210$ \\
Mean age (year \pm SD) & $46.16 \pm 13.09$ & $46.43 \pm 14.58$ \\
Mean age of onset of AD (year \pm SD) & $30.13 \pm 11.49$ & $\mathrm{NA}$ \\
\hline
\end{tabular}

$\leqslant 30$ days before enrollment; and mandated incarceration or employment loss for not receiving alcohol treatment were excluded. All control participants were selected from a large genetic study on tobacco smoking to match ethnicity and age of the $\mathrm{AD}$ participants, and were recruited primarily from the Mid-South States during 1999-2005 (Beuten et al, 2005; Li et al, 2006). All control individuals were screened for a history of substance use disorders and other Axis I psychiatric disorders, and participants who were positive were excluded. Additionally, in the control group, none of the participants reported having consumed greater than four standard drinks in total, during the 90 days before enrollment. Self-reported ethnicity data was collected for both control and $\mathrm{AD}$ individuals at study entry. The demographic characteristics of both $\mathrm{AD}$ and control participants are presented in Table 1.

\section{DNA Extraction, SNP Selection, and Genotyping}

A total of $10 \mathrm{ml}$ of blood was drawn from each participant to obtain white blood cells. The DNA was extracted using a Gentra Puregene kit (QIAGEN, Valencia, CA). We selected 11 single-nucleotide polymorphisms (SNPs) from the National Center for Biotechnology Information (NCBI) dbSNP database (http://www.ncbi.nlm.nih.gov/SNP/) on the basis of high heterozygosity (minor allele frequency $\geqslant 0.05$ ) and chromosomal position for maximum uniform coverage of the gene. Detailed information on SNP locations, chromosomal positions, allelic variants, minor allele frequency, and primer/probe sequences is summarized in Table 2. In addition, 24 Ancestry Informative Markers (AIMs) were genotyped in all $\mathrm{AD}$ and in control participant DNA samples to test for population stratification. The primary reasons for us to use these AIMs are that they have been demonstrated to have high-frequency differences for South American/European ancestry and European/West African ancestry based on a survey study for a genome-wide ancestry marker panel, and are suggested to be essential markers for population stratification investigation (Mao et al, 2007). Information on these marker SNPs are given in Supplementary Table 1.

All SNP assays were carried out with the TaqMan SNP genotyping assay in the 384-well microplate format (Applied Biosystems, Foster City, CA). PCR conditions were $50^{\circ} \mathrm{C}$ for $2 \mathrm{~min}, 95^{\circ} \mathrm{C}$ for $10 \mathrm{~min}, 30$ cycles of $95^{\circ} \mathrm{C}$ for $25 \mathrm{~s}$, and $60^{\circ} \mathrm{C}$ for $1 \mathrm{~min}$. Each reaction was carried out with $10 \mathrm{ng}$ of DNA from each participant, and SNP-specific control samples were added to each 384-well plate to ensure the quality of the assays. Allelic discrimination analyses were performed with an ABI PRISM 7900HT instrument (Applied Biosystems) using sequence detection software.

\section{Statistical Analysis}

We assessed linkage disequilibrium (LD) and haplotype blocks with all 11 SNPs in cases and controls separately, using Haploview (v. 4.1) (Gabriel et al, 2002). The associations between individual SNPs and AD were analyzed with Unphased (v. 3.0.13) software (Dudbridge, 2008), which calculates a maximum likelihood ratio statistic allowing for covariate adjustments. An association of a SNP with $\mathrm{AD}$ was considered significant only if its $p$ value was 
Table 2 Information on NKIR SNPs Genotyped in the Current Study

\begin{tabular}{|c|c|c|c|c|c|}
\hline SNP number & dbSNP ID & Location within the gene & Physical location & Alleles & ABI SNP assay ID \\
\hline । & rs21।I375 & Intergenic at $5^{\prime}$ end & 54243815 & $\mathrm{G} / \mathrm{A}$ & C_2679170_10 \\
\hline 2 & rs67I5729 & Silent mutation on Exon I & $5424 \mid 665$ & G/A & C_25473413_10 \\
\hline 3 & rs377। 860 & Intron I & 54231673 & $\mathrm{~T} / \mathrm{G}$ & C_275I4344_10 \\
\hline 4 & rs3821319 & Intron I & 54205876 & $C / G$ & C_26138472_10 \\
\hline 5 & rs735668 & Intron I & $54|7898|$ & $\mathrm{C} / \mathrm{A}$ & C_2679|14_10 \\
\hline 8 & rs4439987 & Intron 2 & 54103043 & $A / G$ & C_1227902_10 \\
\hline 9 & rs $727 \mid 56$ & Intron 3 & $54096 \mid 44$ & $\mathrm{~T} / \mathrm{C}$ & C_580584_10 \\
\hline 10 & rs|lo6855 & Intron 4 & 54093924 & $\mathrm{~A} / \mathrm{G}$ & C_1227897_10 \\
\hline । & rs88। & $3^{\prime}-$ UTR & 54092366 & $C / G$ & C_II466393_10 \\
\hline
\end{tabular}

less than the corrected $p$ value based on the SNP spectral decomposition (SNPSpD) approach for multiple testing (Nyholt, 2004). As calculated by SNPSpD, the corrected statistically significant $p$ value for 11 SNP analyses was set at $p=0.006$. The associations between haplotypes and $\mathrm{AD}$ were analyzed with Haplo Stats (v. 1.2.1) software (http:// mayoresearch.mayo.edu/mayo/research/biostat/schaid.cfm). We defined a 'major haplotype' as one with a frequency of more than $5 \%$ in either the $\mathrm{AD}$ or the control samples. Bonferroni correction was used to calculate the corrected $p$ value for the haplotype analyses. In both individual SNP and haplotype association analyses with $\mathrm{AD}$, using Unphased and Haplo Stats programs, respectively, age and sex were used as modifiers, and site Ids, where each $\mathrm{AD}$ and control participant was recruited initially, were used as confounders in all our association analyses. Furthermore, to test for possible moderating effects of each covariate on the observed associations between $\mathrm{AD}$ and individual SNPs, we employed a general linear model (GLM) in SAS (v. 9.1) (SAS Institute Inc, 2003) that included age, sex, and center IDs as main effects as well as their interactions with individual SNPs.

The program Structure 2.2 (http://pritch.bsd.uchicago. edu/software/structure2_2.html) was used to assess the population stratification in the participants enrolled at the three sites. Simulation parameters were set to 10000 burnins, 10000 iterations, and $K$ was set to 3 (for three recruitment sites).

\section{RESULTS}

\section{Individual SNP Association Analyses with AD}

Genotypic distributions of all 11 SNPs conformed to the Hardy-Weinberg equilibrium with the smallest $p$ value being 0.102 . The genotypic and allelic frequencies for each SNP in both case and control samples are shown in Table 3. Individual SNP-based association analyses performed with Unphased (v. 3.0.13) software, using a statistical model incorporating age, sex, and site IDs as covariates, revealed that the genotypic and allelic frequencies were significantly different in the case and the control samples for SNPs, rs2111375 $(p=0.0454)$ and rs6715729 $(p=0.0012)$. After correction for multiple testing, only SNP rs6715729 remained significant. When the frequency of the $G$ allele, which is the minor allele for rs6715729 in the AD sample, was compared with the frequency of the $G$ allele in the control group, the difference was statistically significant $(p=0.0006)$ with an odds ratio (OR) of $6.13(95 \% \mathrm{CI}=4.06$, 9.23). As revealed by SAS GLM model, the interaction between rs6715729 and site IDs was significant at $p<0.0001$; notably, the individual SNP rs6715729 effect on AD also remained significant $(p=0.0002)$ in this GLM model, confirming the genetic effect of rs6715729 on AD.

\section{Haplotype Analyses}

We employed Haploview to calculate pair-wise correlation coefficients $\left(r^{2}\right.$, the squared total LD divided by the product of the allelic frequencies at both loci) to detect haplotype blocks among all 11 SNPs according to the criteria defined by Gabriel et al (2002). As shown in Figure 1, we detected three haplotype blocks in the control sample. These blocks, each comprising two-SNP combinations, were located at the $5^{\prime}$ end of the gene, around exon 2, and at the $3^{\prime}$ end of the gene: rs2111375-rs6715729, $1 \mathrm{~kb}$; rs735668-rs6741029, $17 \mathrm{~kb}$; rs1106855-rs881, $2 \mathrm{~kb}$. In contrast, we did not detect any haplotype blocks in the $\mathrm{AD}$ sample.

Next, using Haplo Stats, we performed haplotype-based association analyses in several ways: First, within each Haploview-defined LD block comprising of two SNPs, as shown in Figure 1; and second, with all possible three-SNP combinations within the three Haploview-defined LD blocks. In addition, we performed a haplotype analysis with all 27 possible three-SNP haplotype combinations formed by the 11 SNPs that were genotyped in this study. The haplotype analyses within the three two-SNP LD blocks, detected only one Haploview-defined LD block (rs735668 and rs6741029) to be significantly associated with $\mathrm{AD}$ (global association test $p=1.439 \times 10^{-9}$ ). Our second approach, the haplotype analyses with all six SNPs within the three Haploview-defined LD blocks, yielded four major haplotypes formed by a three-SNP combination of rs6715729-rs7356680-rs6741029 (Table 4). These three SNPs were located in adjacent LD blocks within a distance of $80533 \mathrm{bp}$ in the NK1R gene, and comprised of SNP rs6715729 that was significantly associated with $\mathrm{AD}$ at 
Table 3 Genotype and Allele Frequencies of NKIR SNPs for Alcohol-Dependent and Control Participants

\begin{tabular}{|c|c|c|c|c|c|c|c|c|c|c|c|c|c|c|}
\hline \multirow[t]{3}{*}{ db SNP ID } & \multicolumn{2}{|c|}{$\begin{array}{c}\text { Frequencies from } \\
\text { HapMap-CEU data set }\end{array}$} & \multicolumn{7}{|c|}{$\begin{array}{l}\text { Genotype count (frequency) } \\
\text { from the present study }\end{array}$} & \multicolumn{5}{|c|}{ Allele count (frequency) } \\
\hline & \multirow[t]{2}{*}{ Genotype } & \multirow[t]{2}{*}{ Allele } & \multicolumn{3}{|c|}{$\mathbf{A D}$} & \multicolumn{3}{|c|}{ Controls } & \multirow{2}{*}{ P-value* } & \multicolumn{2}{|c|}{$\mathbf{A D}$} & \multicolumn{2}{|c|}{ Controls } & \multirow[t]{2}{*}{$P$-value** } \\
\hline & & & $1 / 1$ & $1 / 2$ & $2 / 2$ & $1 / 1$ & $1 / 2$ & $2 / 2$ & & $\mathbf{I}$ & 2 & $\mathbf{I}$ & 2 & \\
\hline $\begin{array}{l}\text { rs } 2 \text { II I I375 } \\
I=G \\
2=A\end{array}$ & $\begin{array}{l}G G=0.518 \\
G A=0.393 \\
A A=0.089\end{array}$ & $\begin{array}{l}G=0.7 \mid 4 \\
A=0.286\end{array}$ & $147(0.553)$ & $102(0.383)$ & $17(0.064)$ & I $83(0.555)$ & $126(0.382)$ & $21(0.064)$ & 0.0454 & $396(0.744)$ & $136(0.256)$ & $492(0.745)$ & $168(0.255)$ & 0.0155 \\
\hline $\begin{array}{l}r s 67 \mid 5729 \\
I=G \\
2=A\end{array}$ & $\begin{array}{l}G G=0.130^{\mathrm{a}} \\
\mathrm{GA}=0.391 \\
\mathrm{AA}=0.478\end{array}$ & $\begin{array}{l}G=0.326^{a} \\
A=0.674\end{array}$ & $63(0.238)$ & $129(0.487)$ & $73(0.275)$ & $92(0.277)$ & $164(0.494)$ & $76(0.229)$ & 0.0012 & $255(0.481)$ & $275(0.519)$ & $348(0.524)$ & $316(0.476)$ & 0.0006 \\
\hline $\begin{array}{l}\text { rs } 377 / 1860 \\
I=T \\
2=G\end{array}$ & $\begin{array}{l}T=0.491 \\
T G=0.404 \\
G G=0.105\end{array}$ & $\begin{array}{l}T=0.693 \\
G=0.307\end{array}$ & $153(0.584)$ & 91 (0.347) & $18(0.069)$ & | 85 (0.556) & $132(0.396)$ & $16(0.048)$ & 0.4853 & $397(0.758)$ & $127(0.242)$ & $502(0.754)$ & $164(0.246)$ & 0.2544 \\
\hline $\begin{array}{l}r s 3821319 \\
I=C \\
2=G\end{array}$ & $\begin{array}{l}C C=0.186 \\
C G=0.475 \\
G G=0.339\end{array}$ & $\begin{array}{l}C=0.424 \\
G=0.576\end{array}$ & $30(0.113)$ & $98(0.370)$ & $137(0.5 \mid 7)$ & $50(0.150)$ & I $48(0.443)$ & $136(0.407)$ & 0.6243 & I $58(0.298)$ & $372(0.702)$ & $248(0.37 \mid)$ & $420(0.629)$ & 0.4708 \\
\hline $\begin{array}{l}r s 735668 \\
I=C \\
2=A\end{array}$ & $\begin{array}{l}C C=0.281 \\
C A=0.474 \\
A A=0.246\end{array}$ & $\begin{array}{l}C=0.518 \\
A=0.482\end{array}$ & $73(0.277)$ & $123(0.466)$ & $68(0.258)$ & $77(0.230)$ & $165(0.493)$ & $93(0.278)$ & 0.2129 & $269(0.509)$ & $259(0.491)$ & $319(0.476)$ & $351(0.524)$ & 0.1393 \\
\hline $\begin{array}{l}r s 6741029 \\
I=G \\
2=T\end{array}$ & $\begin{array}{l}\mathrm{GG}=0.458 \\
\mathrm{GT}=0.373 \\
\Pi T=0.169\end{array}$ & $\begin{array}{l}G=0.644 \\
T=0.356\end{array}$ & $100(0.392)$ & $112(0.439)$ & $43(0.169)$ & $138(0.4 \mid 4)$ & I $54(0.462)$ & $41(0.123)$ & 0.3023 & $312(0.611)$ & $198(0.388)$ & $430(0.646)$ & $236(0.354)$ & 0.2172 \\
\hline $\begin{array}{l}r s 4853105 \\
I=T \\
2=A\end{array}$ & $\begin{array}{l}T=0.167 \\
T A=0.333 \\
A A=0.500\end{array}$ & $\begin{array}{l}T=0.333 \\
A=0.667\end{array}$ & $32(0.122)$ & $102(0.389)$ & $128(0.489)$ & $34(0.103)$ & $149(0.450)$ & | $48(0.447)$ & 0.1212 & $166(0.317)$ & $358(0.683)$ & $217(0.328)$ & $445(0.672)$ & 0.0665 \\
\hline $\begin{array}{l}r s 4439987 \\
I=G \\
2=A\end{array}$ & $\begin{array}{l}G G=0.220 \\
G A=0.542 \\
A A=0.237\end{array}$ & $\begin{array}{l}G=0.492 \\
A=0.508\end{array}$ & $58(0.229)$ & $133(0.526)$ & $62(0.245)$ & $69(0.208)$ & $142(0.428)$ & $121(0.364)$ & 0.4375 & $249(0.492)$ & $257(0.508)$ & $280(0.422)$ & $384(0.578)$ & 0.3739 \\
\hline $\begin{array}{l}r s 727 \mid 56 \\
I=T \\
2=C\end{array}$ & $\begin{array}{l}T=0.768 \\
T C=0.196 \\
C C=0.036\end{array}$ & $\begin{array}{l}T=0.866 \\
C=0.134\end{array}$ & | $87(0.7||)$ & $67(0.255)$ & $9(0.034)$ & $219(0.662)$ & $96(0.290)$ & $16(0.048)$ & 0.7694 & $44 \mid(0.838)$ & $85(0.162)$ & $534(0.807)$ & $128(0.193)$ & 0.7225 \\
\hline $\begin{array}{l}r s \mid l 06855 \\
I=G \\
2=A\end{array}$ & $\begin{array}{l}G G=0.593 \\
A G=0.339 \\
A A=0.068\end{array}$ & $\begin{array}{l}G=0.763 \\
A=0.237\end{array}$ & I 49 (0.573) & $90(0.346)$ & $21(0.08 \mid)$ & $196(0.585)$ & II (0.349) & $22(0.066)$ & 0.8343 & $388(0.746)$ & $132(0.254)$ & $509(0.760)$ & $|6|(0.240)$ & 0.8255 \\
\hline $\begin{array}{l}r s 88 I \\
I=G \\
2=C\end{array}$ & $\begin{aligned} G G & =0.719 \\
G C & =0.263 \\
C C & =0.018\end{aligned}$ & $\begin{array}{l}G=0.85 I \\
C=0.149\end{array}$ & I 84 (0.727) & $59(0.233)$ & $10(0.040)$ & $221(0.664)$ & $93(0.279)$ & $19(0.057)$ & 0.9394 & $427(0.844)$ & $79(0.156)$ & $535(0.803)$ & $|3|(0.197)$ & 0.7564 \\
\hline
\end{tabular}



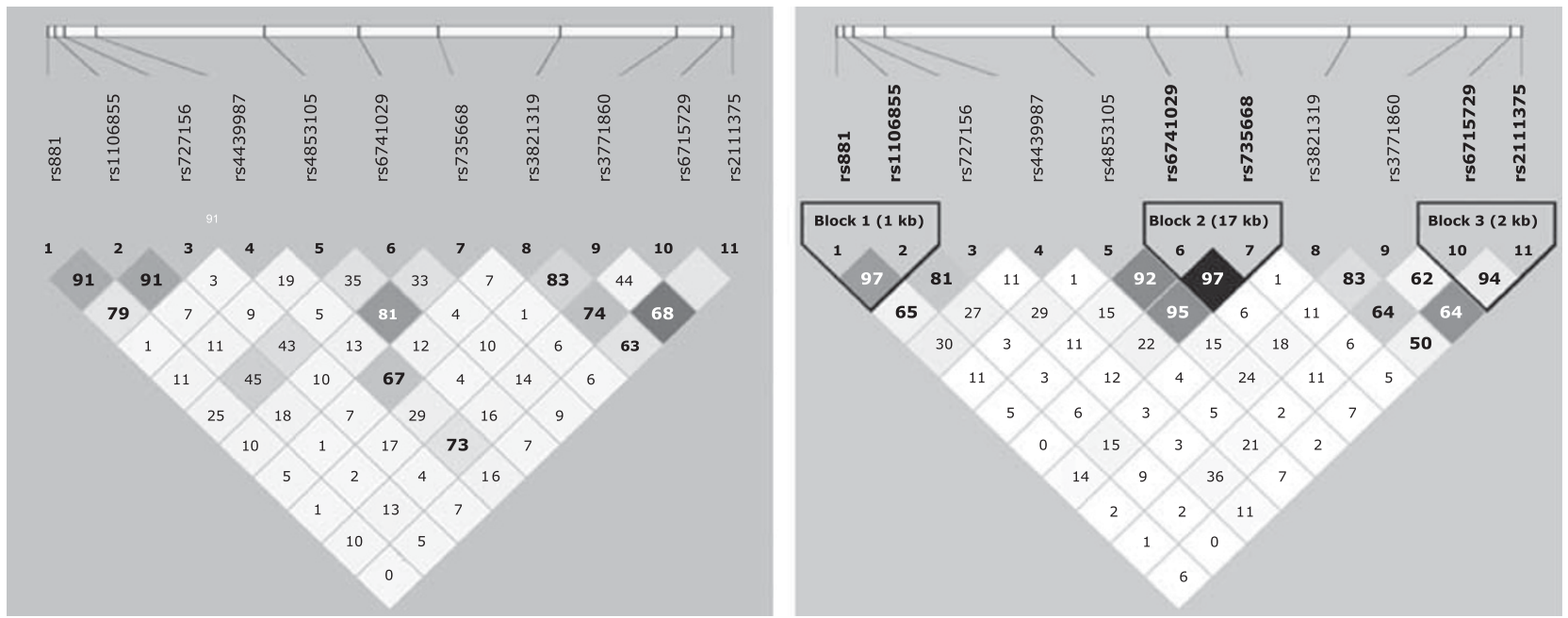

Figure I Linkage disequilibrium analysis of NKIR SNPs in Caucasian alcohol-dependent (AD) and control samples. Values in squares are $r^{2}$ numbers.

Table 4 Association Results of Major Haplotypes Formed by SNPs, rs67I5729, rs735668, and rs674I029, with AD

\begin{tabular}{|c|c|c|c|c|c|c|c|c|}
\hline \multirow[t]{2}{*}{ Haplotype } & \multirow[t]{2}{*}{ Haplo-score } & \multirow[t]{2}{*}{$P$-value } & \multicolumn{3}{|c|}{ Haplotype frequency } & \multicolumn{3}{|c|}{ Odds ratio } \\
\hline & & & Pooled & Controls & AD & Lower OR & OR & Upper OR \\
\hline G-A-G & -2.3559 & 0.0184 & 0.0737 & 0.0995 & 0.0478 & 0.3415 & 0.6192 & 1.1227 \\
\hline$A-A-T$ & -1.5829 & 0.1134 & 0.1301 & 0.1598 & 0.0921 & 0.4229 & 0.7184 & 1.2203 \\
\hline$A-A-G$ & 2.8953 & 0.0038 & 0.1267 & 0.0805 & 0.1846 & 1.1552 & 1.8938 & 3.1045 \\
\hline G-C-T & 6.0062 & $1.8992 \times 10^{-9}$ & 0.0486 & 0.0075 & 0.1092 & 3.6194 & 11.3113 & 35.3498 \\
\hline \multicolumn{9}{|c|}{ Global-stat $=59.2790 ;$ d.f. $=7 ; p$-value $=2.1024 \times 10^{-10}$} \\
\hline
\end{tabular}

Statistically significant $P$-values (after correcting for multiple testing) for individual haplotype frequency differences in AD and control sample are shown in bold italics.

individual level. Therefore, we considered three-SNP combination formed by rs6715729-rs7356680-rs6741029 to be more informative than the two-SNP combination formed by rs7356680 and rs6741029 alone. Of the four major haplotypes formed by rs6715729-rs7356680rs6741029, two were negatively associated with AD (G-CG, G-A-G), whereas the other two were positively associated with AD (A-A-G, G-C-T). After correcting for multiple testing, only the two positively associated haplotypes remained significant. (The corrected statistically significant $p$ value for a three-SNP haplotype analysis was set at $p=0.006$.) The A-A-G and G-C-T haplotypes together accounted for $17.5 \%$ of the pooled sample $(29.4 \%$ of the $\mathrm{AD}$ sample and $8.8 \%$ of the control sample). The observed frequency differences for haplotypes A-A-G $(18.5 \%$ in $\mathrm{AD}$ and $8.1 \%$ in controls) and G-C-T (10.9\% in AD and $0.8 \%$ in controls) in the $\mathrm{AD}$ and control samples were significantly different $\left(\chi^{2}=2.8953, p=0.0038\right.$ and $\chi^{2}=6.0062$, $p=1.8992 \times 10^{-9}$, respectively) with corresponding ORs of $1.89(95 \% \mathrm{CI}=1.16,3.11)$ for $\mathrm{A}-\mathrm{A}-\mathrm{G}$ and $11.31(95 \%$ $\mathrm{CI}=3.62,35.35)$ for G-C-T. Therefore, the odds of becoming dependent on alcohol were more than one in participants carrying either of these two haplotypes. Hence, they were named 'risk haplotypes.' The SNPs that formed the two risk haplotypes were located in exon 1 and in the intronic regions on either side of exon 2 spanning about $82 \mathrm{~kb}$.

Population stratification for participants enrolled at the three study sites was assessed with 24 AIM SNP markers. Results from program Structure showed no evidence for the presence of population admixture in both case and control participants included in this study (see Supplementary Figure 1). Therefore, all 608 participants were included in association analyses with $\mathrm{AD}$, both at individual and haplotype levels.

\section{DISCUSSION}

In this study, we provide the first evidence for an association of NK1R polymorphisms with $\mathrm{AD}$ in Caucasian individuals. After genotyping for 11 SNPs spanning the 
gene, we detected SNP, rs6715729, and two haplotypes, A-A$\mathrm{G}$ and G-C-T, formed by rs6715729-rs7356680-rs6741029, that were significantly associated with $\mathrm{AD}$. The allele frequencies of rs6715729, a synonymous SNP in exon 1, differed significantly in the AD and in the control samples. In the AD sample, allele A of rs6715729 was significantly more frequent than that in the control sample. The frequency of the A allele in our control sample was in agreement with the findings of an independent study by Randolph et al (2004) that showed the A allele of rs6715729 to be less frequent than the $G$ allele in a sample of 100 healthy individuals of Caucasian descent.

Moreover, we detected two haplotypes, G-C-T and A-A-G, formed by rs6715729-rs735668-rs6741029, that were significantly associated with $\mathrm{AD}$; and both showed a significant risk effect on the development of $\mathrm{AD}$. The first risk haplotype, $\mathrm{A}-\mathrm{A}-\mathrm{G}$, was twofold more common $(\mathrm{OR}=1.89$; $95 \% \mathrm{CI}=1.16,3.11)$ in the $\mathrm{AD}$ than in the control sample. The second risk haplotype, G-C-T, was present predominantly in the AD sample $(10.92 \%$ compared with $0.75 \%$ of the control sample) with a likelihood of about 11-fold higher in $\mathrm{AD}$ participants than in controls $(\mathrm{OR}=11.31 ; 95 \%$ CI: 3.62, 35.35). By comparing allele composition in the two haplotypes, we found that unlike the A-A-G haplotype, the G-C-T risk haplotype consisted of G allele at rs6715729, which showed a negative association with $\mathrm{AD}$ individually. On the other hand, the other two SNPs in the two haplotypes, rs735668 and rs6741029, did not show a significant association with $\mathrm{AD}$ individually. This suggests that the risk effects of these two haplotypes are conferred by a combination of alleles among the three SNPs. Indeed, haplotype analyses identify a combination(s) of SNPs that modulate each others' individual effects to shape the overall modest effects caused by single genes in the etiology of complex traits such as $\mathrm{AD}$ (Zhang et al, 2008). In this context, another possibility is the presence of an unknown functional cis-acting polymorphism(s) in the region extending from SNPs rs735668 to rs6741029 that is associated with AD.

To our knowledge, this is the first study to investigate associations between $\mathrm{AD}$ and NK1R SNPs. The strength of our findings is enhanced by the differences in LD structures in the cases and controls. In the control sample, the SNPs that constitute the two risk haplotypes were in adjacent haplotype blocks; interestingly, these blocks were not seen in the AD sample, suggesting that this region may have undergone selection. Furthermore, the region of NK1R extending from rs6715729 to rs6741029 corresponds to the $5^{\prime}$ end of NK1R primary mRNA, which is translated into the $\mathrm{N}$-terminal region through the end of the second extracellular loop of NK1 receptors (Almeida et al, 2004). The NK1 receptors are seven-transmembrane domain G-protein coupled with three extracellular loops, three intracellular loops, an intracellular $\mathrm{C}$ terminus, and an extracellular $\mathrm{N}$ terminus. Among the many properties of the $5^{\prime}$ end portion of NK1 receptors important in SP signal transduction, the presence of a binding site for SP has been reported involving the second extracellular loop (Lequin et al, 2002). Although none of the SNPs included in the two risk haplotypes affect the amino acid sequence of a protein, they could affect SP signal transduction by altering receptor density through alternative splicing of NK1R mRNA. Given the large size of the gene and the relatively low density of the SNPs that we have genotyped, future genotyping studies should be aimed at identifying causative loci within this region.

There are several limitations to this study. First, the controls and cases were recruited at three geographic locations. To reduce the potential geographic effects on our association results, we included center ID as a covariate in all the association analyses. In addition, we performed an analysis for genetic structure with 24 unlinked AIMs to exclude ethnic admixture within the Caucasian sample studied. Although the number of AIMs genotyped in our study was not as large as we wish, we do think they are sufficient for our purpose here. This is because: (1) 24 AIMs have been reported to be as effective in estimating continental population ancestry as identified with more than 90 AIMs (Kosoy et al, 2008) and (2) self-reported ethnicity usually is consistent with classification based on molecular markers (Yang et al, 2005). The second main limitation in our study is that the control sample had a relatively higher female population than the $\mathrm{AD}$ sample. This issue was addressed by including sex as a covariate in all the analyses. Third, our cases were a population of $\mathrm{AD}$ individuals who were motivated to decrease or cease their alcohol consumption. Therefore, this cohort may be more motivated and perhaps less dense in drinking pathophysiology than $\mathrm{AD}$ individuals derived from community samples (Wrase et al, 2006).

Numerous studies have affirmed that the product of $N K 1 R$, the receptor, together with its substrate SP, modulates mesocorticolimbic dopamine release, which is essential for the reward involved in AD (Nikolaev et al, 2003; Goodman, 2008). The SP-receptor system is also implicated in the etiology of other psychiatric disorders such as anxiety, depression, or dysfunctional behavioral patterns observable before the onset of AD (Goodman, 2008). Although NK1Rs play a vital role in the etiology of these psychiatric disorders that are comorbid with $\mathrm{AD}$, to date, association studies on NK1R polymorphisms with psychiatric disorders in general are sparse. In a cohort of German participants, Giegling et al (2007) showed that four SNPs located across NK1R were not associated with suicidal behavior, but may modulate aggression features. Nominal associations were found with panic disorder (Hodges et al, 2009) and bipolar disorder (Perlis et al, 2008); lack of an association of NK1R SNPs was reported with autism (Marui et al, 2007) and dyslexia (Peyrard-Janvid et al, 2004). To our knowledge, associations of NK1R polymorphisms with any substance use disorders have not been examined.

In summary, we detected SNP, rs6715729, and two haplotypes in $N K 1 R$ that were significantly associated with $\mathrm{AD}$ in Caucasians. These results strongly indicate that NK1R is involved in the etiology of AD. In consideration of the relatively small sample size, more replication is greatly needed in a larger sample of both Caucasian and other ethnic samples. Furthermore, more molecular studies are needed to identify the mechanism(s) by which these genetic variants affect the development of $\mathrm{AD}$.

\section{ACKNOWLEDGEMENTS}

This study was funded by grants from the National Institute on Alcohol Abuse and Alcoholism to BAJ (Grants 7 U10 
AA011776-10, 1 N01 AA001016-000, 7 R01 AA010522-12, and 5 R01 AA012964-06) and NA-D (Grant 5 K23 AA000329-06) and from the National Institute on Drug Abuse to MDL (Grants R01 DA012844 and 5 R01 DA013783). We appreciate the skilled technical assistance of the staff at the South Texas Addiction Research and Technology Center, Department of Psychiatry, The University of Texas Health Science Center at San Antonio, and the Department of Psychiatry and Neurobehavioral Sciences, University of Virginia. We also are grateful to Dr Xiang-Yang Lou for his expert advice and Eva JenkinsMendoza, BS, for her service as project coordinator.

\section{DISCLOSURE/CONFLICT OF INTEREST}

Related to this report, authors Seneviratne, Ait-Daoud, Ma, Chen and Li have nothing to disclose. Professor Johnson currently serves as a consultant to Johnson \& Johnson (Ortho-McNeil Janssen Scientific Affairs, LLC), Transcept Pharmaceuticals, Organon, ADial Pharmaceuticals, and Eli Lilly and Company.

\section{REFERENCES}

Almeida TA, Rojo J, Nieto PM, Pinto FM, Hernandez M, Martin JD et al (2004). Tachykinins and tachykinin receptors: structure and activity relationships. Curr Med Chem 11: 2045-2081.

American Psychiatric Association (1994). Diagnostic and Statistical Manual of Mental Disorders, 4th edn. American Psychiatric Association: Washington, DC.

Babor TF, de la Fuente JR, Saunders J, Grant M (1992). AUDIT: The Alcohol Use Disorders Identification Test. World health organization: Geneva, Switzerland.

Beuten J, Ma JZ, Payne TJ, Dupont RT, Crews KM, Somes G et al (2005). Single- and multilocus allelic variants within the GABAB receptor subunit 2 (GABAB2) gene are significantly associated with nicotine dependence. Am J Hum Genet 76: 859-864.

Caberlotto L, Hurd YL, Murdock P, Wahlin JP, Melotto S, Corsi M et al (2003). Neurokinin 1 receptor and relative abundance of the short and long isoforms in the human brain. Eur J Neurosci 17: 1736-1746.

Dudbridge F (2008). Likelihood-based association analysis for nuclear families and unrelated subjects with missing genotype data. Hum Hered 66: 87-98.

First MB, Spitzer RL, Gibbon M, Williams JBW (1994). Structured Clinical Interview for DSM-IV Axis I Disorders-Patient Edition (SCID-I/P version 2.0). New York State Psychiatric Institute, Biometrics Research Department: New York.

Gabriel SB, Schaffner SF, Nguyen H, Moore JM, Roy J, Blumenstiel $B$ et al (2002). The structure of haplotype blocks in the human genome. Science 296: 2225-2229.

Garbutt JC, Kranzler HR, O'Malley SS, Gastfriend DR, Pettinati HM, Silverman BL et al (2005). Efficacy and tolerability of longacting injectable naltrexone for alcohol dependence: a randomized controlled trial. JAMA 293: 1617-1625.

George DT, Gilman J, Hersh J, Thorsell A, Herion D, Geyer C et al (2008). Neurokinin 1 receptor antagonism as a possible therapy for alcoholism. Science 319: 1536-1539.

Giegling I, Rujescu D, Mandelli L, Schneider B, Hartmann AM, Schnabel A et al (2007). Tachykinin receptor 1 variants associated with aggression in suicidal behavior. Am J Med Genet B Neuropsychiatr Genet 144B: 757-761.

Goodman A (2008). Neurobiology of addiction. An integrative review. Biochem Pharmacol 75: 266-322.
Hodges LM, Weissman MM, Haghighi F, Costa R, Bravo O, Evgrafov $\mathrm{O}$ et al (2009). Association and linkage analysis of candidate genes GRP, GRPR, CRHR1, and TACR1 in panic disorder. Am J Med Genet B Neuropsychiatr Genet 150B: 65-73.

Holmes A, Heilig M, Rupniak NM, Steckler T, Griebel G (2003). Neuropeptide systems as novel therapeutic targets for depression and anxiety disorders. Trends Pharmacol Sci 24: 580-588.

Johnson BA (2008). Update on neuropharmacological treatments for alcoholism: scientific basis and clinical findings. Biochem Pharmacol 75: 34-56.

Kosoy R, Nassir R, Tian C, White PA, Butler LM, Silva G et al (2008). Ancestry informative marker sets for determining continental origin and admixture proportions in common populations in america. Hum Mutat 30: 69-78.

Lai JP, Cnaan A, Zhao H, Douglas SD (2008). Detection of fulllength and truncated neurokinin-1 receptor mRNA expression in human brain regions. J Neurosci Methods 168: 127-133.

Lequin O, Bolbach G, Frank F, Convert O, Girault-Lagrange S, Chassaing $\mathrm{G}$ et al (2002). Involvement of the second extracellular loop (E2) of the neurokinin-1 receptor in the binding of substance P. Photoaffinity labeling and modeling studies. J Biol Chem 277: 22386-22394.

Li MD, Payne TJ, Ma JZ, Lou XY, Zhang D, Dupont RT et al (2006). A genomewide search finds major susceptibility loci for nicotine dependence on chromosome 10 in African Americans. Am J Hum Genet 79: 745-751.

Mao X, Bigham AW, Mei R, Gutierrez G, Weiss KM, Brutsaert TD et al (2007). A genomewide admixture mapping panel for Hispanic/Latino populations. Am J Hum Genet 80: 1171-1178.

Marui T, Funatogawa I, Koishi S, Yamamoto K, Matsumoto H, Hashimoto O et al (2007). Tachykinin 1 (TAC1) gene SNPs and haplotypes with autism: a case-control study. Brain Dev 29: 510-513.

Nikolaev SV, Bychkov ER, Lebedev AA, Dambinova SA (2003). Mechanisms of the influences of the central administration of substance $\mathrm{P}$ on ethanol consumption in chronically alcoholic rats. Neurosci Behav Physiol 33: 905-909.

Nyholt DR (2004). A simple correction for multiple testing for single-nucleotide polymorphisms in linkage disequilibrium with each other. Am J Hum Genet 74: 765-769.

Perlis RH, Purcell S, Fagerness J, Kirby A, Petryshen TL, Fan J et al (2008). Family-based association study of lithium-related and other candidate genes in bipolar disorder. Arch Gen Psychiatry 65: $53-61$.

Peyrard-Janvid M, Anthoni H, Onkamo P, Lahermo P, Zucchelli M, Kaminen $\mathrm{N}$ et al (2004). Fine mapping of the $2 \mathrm{p} 11$ dyslexia locus and exclusion of TACR1 as a candidate gene. Hum Genet 114: 510-516.

Randolph GP, Simon JS, Arreaza MG, Qiu P, Lachowicz JE, Duffy RA (2004). Identification of single-nucleotide polymorphisms of the human neurokinin 1 receptor gene and pharmacological characterization of a Y192H variant. Pharmacogenomics J 4: 394-402.

Santarelli L, Gobbi G, Debs PC, Sibille ET, Blier P, Hen R et al (2001). Genetic and pharmacological disruption of neurokinin 1 receptor function decreases anxiety-related behaviors and increases serotonergic function. Proc Natl Acad Sci USA 98: 1912-1917.

SAS Institute Inc (2003). SAS Version 9.1. SAS Institute Inc.: Cary, NC.

Sinha R, Robinson J, O’Malley S (1998). Stress response dampening: effects of gender and family history of alcoholism and anxiety disorders. Psychopharmacology (Berl) 137: 311-320.

Sullivan JT, Sykora K, Schneiderman J, Naranjo CA, Sellers EM (1989). Assessment of alcohol withdrawal: the revised clinical institute withdrawal assessment for alcohol scale (CIWA-Ar). $\mathrm{Br}$ J Addict 84: 1353-1357. 
Wrase J, Reimold M, Puls I, Kienast T, Heinz A (2006). Serotonergic dysfunction: brain imaging and behavioral correlates. Cogn Affect Behav Neurosci 6: 53-61.

Yang BZ, Kranzler HR, Zhao H, Gruen JR, Luo X, Gelernter J (2007). Association of haplotypic variants in DRD2, ANKK1, TTC12 and NCAM1 to alcohol dependence in independent case control and family samples. Hum Mol Genet 16: 2844-2853.
Yang BZ, Zhao H, Kranzler HR, Gelernter J (2005). Practical population group assignment with selected informative markers: characteristics and properties of Bayesian clustering via STRUCTURE. Genet Epidemiol 28: 302-312.

Zhang H, Kranzler HR, Yang BZ, Luo X, Gelernter J (2008). The OPRD1 and OPRK1 loci in alcohol or drug dependence: OPRD1 variation modulates substance dependence risk. Mol Psychiatry 13: $531-543$.

Supplementary Information accompanies the paper on the Neuropsychopharmacology website (http://www.nature.com/npp) 